\title{
La vérité sur la Grande Famine (Ukraine 1931-1934)
}

\author{
Georges NIVAT \\ Professeur honoraire \\ Faculté des Lettres, Département MESLO \\ Université de Genève $(\mathrm{CH})$ \\ Georges.Nivat@unige.ch
}

\section{Doi : 10.5077/journals/connexe.2021.e607}

\section{Résumé}

Partant du film de la réalisatrice polonaise Agnieszka Holland, L'ombre de Staline, cet article est le fruit d'une relecture des textes et carnets de Gareth Jones, un intrépide jeune journaliste gallois qui, en 1931, après avoir interviewé Hitler, se met en tête d’interviewer Staline. Mais, arrivé à Moscou avec une lettre de recommandation de Lloyd George, il découvre, par une collègue, la grande famine en Ukraine, que le régime et la quasi-totalité des journalistes occidentaux en poste en URSS font de leur mieux pour dissimuler.

Les textes et carnets de Gareth révèlent des détails effarants sur cette famine organisée pour pouvoir exporter autant de grain que possible.

L'article étudie l'effet du tabou stalinien relayé par la presse occidentale, après que Jones a vraiment clamé la vérité (et qu'il eut été tué en Mandchourie deux ans plus tard - probablement par vengeance). Puis, cet article montre la lente progression des études sur cet effrayant épisode d'une famine provoquée. Tout d'abord, le camouflage obstiné, mis en place par le journaliste américain et prix Pullizer en 1932, Walter Duranty, sera étudié. Ensuite, la lente avancée des connaissances sur cette période sera examinée, depuis les travaux de Boris Souvarine et son Staline (1935), jusqu'aux recherches de Robert Conquest publiées dans Harvest of Sorrow (1986), pour terminer avec le dernier ouvrage d’Anne Applebaum, Red Famine (2017), qui pose, entre autres, la question : La Grande Famine en Ukraine relève-t-elle du crime de génocide?

Mots-clés : Famine, Ukraine, Gareth Jones, Robert Conquest, Anne Applebaum, génocide.

\section{Abstract}

Starting from the Polish director Agnieszka Holland's film, Mr Jones, The Truth that can't be hidden forever, this article is the result of an extensive reading of the papers and notebooks of Gareth Jones, a valiant young Welsh journalist who, in 1931, after interviewing Hitler, sets out to interview Stalin. But, when he arrived in Moscow, with a recommendation letter from Lloyd George, he discovered, with the help of a colleague's confidence, that a severe famine was occurring in Ukraine, and that the regime and nearly all the Western journalists appointed in the USSR were doing their utmost to conceal it.

Gareth's articles and note-books reveal appalling details of this famine, which was organised to export as much grain as possible.

This article analyses the process of the Stalinian taboo, and its relaying by Western press just after Jones has proclaimed the truth about the Famine, (and, two years later, has been killed in Manchuria, probably as a vengeance). Then, it shows the slow progression of studies on this frightening episode of a provoked famine. First, the obstinate cover-up, set up by the American journalist and Pullizer Prize winner in 1932, Walter Duranty, will be studied. Then, the slow progress of knowledge on this period will be examined, from the work of Boris Souvarine and his Stalin (1935) to the research of Robert Conquest published in Harvest of Sorrow (1986), to end with Anne Applebaum's latest book, Red Famine (2017), which raises the question, among others: Does the Great Famine in Ukraine correspond to the crime of genocide?

Keywords: Famine, Ukraine, Gareth Jones, Robert Conquest, Anne Applebaum, genocide. 


\section{Introduction}

En 1935, à la veille de ses trente ans, Gareth Jones, un journaliste intrépide venu d'un tout petit village du pays de Galles, Barry, au sud de Cardiff, partit par ses propres moyens (il avait toujours été un journaliste free-lance) enquêter sur les atrocités japonaises en Mongolie. Son compagnon de route était un Anglais rencontré sur place. Les deux hommes furent kidnappés par des bandits, Gareth fut gardé en otage, le second relâché. Gareth fut tué deux jours après. Le véhicule qui les avait amenés appartenait au Commissariat du peuple aux Affaires intérieures, le NKVD russe, le compagnon de Gareth probablement aussi. Cette étrange disparition était-elle la vengeance de Staline, qui avait le bras long et jamais ne lâchait ses adversaires ? Une nièce de Gareth, Margaret Siriol Colley, qui entama bien plus tard une enquête, en est persuadée (Gareth Jones: A Manchukuo Incident, 1999). C'est elle qui reçut, en 2008, à Westminster Hall, des mains de l'ambassadeur d'Ukraine, l'Ordre du Mérite ukrainien attribué à titre posthume à son oncle pour avoir tenté d'alerter le monde au sujet de la grande famine de 1932-1934, que les Ukrainiens appellent Holodomor (extermination par la famine). La tentative d'alerter le monde qu'avait faite Gareth Jones avait d'ailleurs été vaine. Un journaliste beaucoup plus connu, Walter Duranty, longtemps en poste à Moscou, et qui y bénéficiait d'une sorte de monopole de l'information accordé par les autorités soviétiques, avait reçu le prix Pulitzer en 1932, après avoir balayé les «mensonges » de Jones dans un article retentissant du New York Times. En fait, Duranty était un affidé des services spéciaux staliniens. Ceux-ci s'étaient mis en branle pour déprécier, dénoncer, voire liquider ceux qui tentaient de faire connaître le désastre, un désastre qui frappait les paysans ukrainiens, mais aussi ceux du Kouban, de la Volga, et d'autres régions de l'URSS.

Car l'Ukraine n'était certes pas le seul territoire de l'URSS où l'obstination forcenée de Staline, renforcée par deux mauvaises récoltes, provoqua une famine cauchemardesque. Récemment, l'écrivaine tatare Güzel' Jahina a donné dans Zouleïkha ouvre les yeux [Зулейха открывает глаза, 2015 (traduction française 2017)] un saisissant tableau de l'organisation de la famine dans la campagne du Tatarstan, et de la déportation des « koulaks » tatars. Lors de la famine en OutreVolga en 1891, Léon Tolstoï et toute sa famille étaient allés sur place organiser les secours. Lors de la famine de 1921, à l'appel de Nadežda Krupskaja, l'épouse de Lénine, la Croix rouge internationale était intervenue. En 1931-1932, ce fut le contraire : interdiction de toute aide, déportations massives des koulaks, suivie d'une interdiction de sortir des zones touchées lorsque la faim réduisit hommes, femmes et enfants à mourir lentement, ou parfois à se transformer en cannibales. C'était l'organisation systématique de ce qu'Ante Ciliga a appelé le «pays du Grand Mensonge ». Mensonge gigantesque, auquel le mythe de la « grande lueur à l'Est » et 
de la fabrique de «l'homme nouveau » conférait vraisemblance, fermant les yeux et les bouches.

\section{La vérité qui ne peut pas être cachée, mais qui le fut ...}

Un superbe film d'Agnieszka Holland, une réalisatrice polonaise auteure de nombreux films à la limite du documentaire et de la fiction, est venu donner, en 2020, une saisissante réalité à ce "mensonge déconcertant », en racontant la tentative du jeune journaliste gallois Gareth Jones de vérifier de ses yeux, puis de dénoncer à la face du monde occidental la famine organisée de 1931-1932.

Le film, intitulé dans sa version originale Mr Jones, The Truth that can't be hidden forever ${ }^{1}$ est un "J'accuse » formidable, qui émerge presque un siècle plus tard, et nous confronte non seulement au cynisme de Staline, à l'asservissement des âmes dans la Russie soviétique de l'époque, mais aussi à l'acquiescement veule de l'Occident. Le sombre coloris sépia du film, les lents travellings sur le visage de Gareth, les accélérés qui soulignent la fuite du train de luxe dont on ferme les volets pour que ses passagers ne voient rien, ou la course à vélo finale de Gareth pour aller au château gallois du magnat américain de la presse américaine, William Randolph Hearst, y pénétrer par effraction et convaincre Hearst de lui donner une chance de publier face au monde entier son témoignage - sur les Terres Noires d'Ukraine ravagées par la faim - ce retour aux gravures sépia d'antan nous prépare à l'intrusion du monstre : la

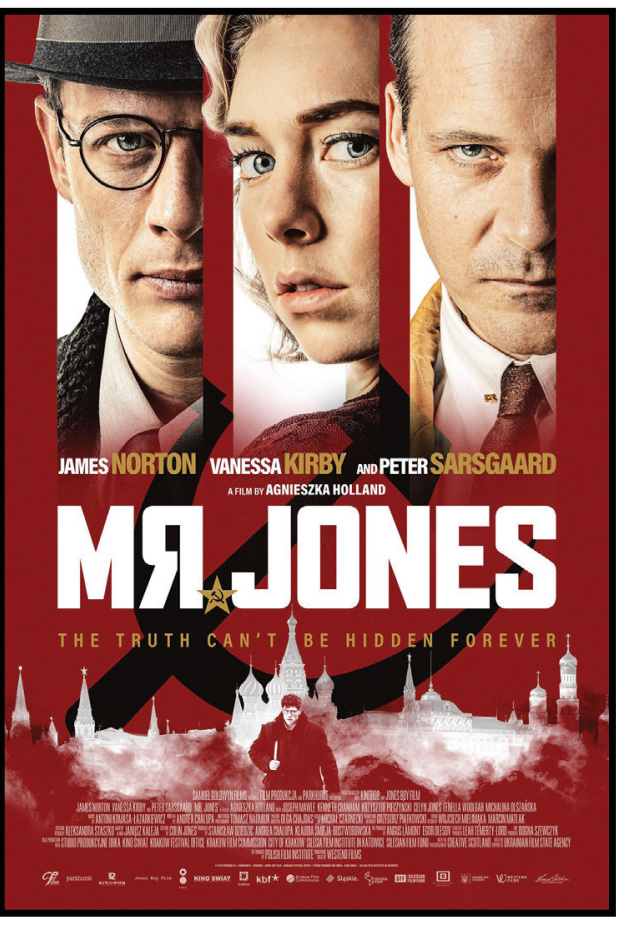
Famine. La Famine que l'écrivain Leonid Andreev (1871-1919) avait, dans un célèbre drame symboliste de 1908, baptisée « Notre Souveraine la Famine » [Царь-Годод, 1908]. Dans la pièce d'Andreev, la Faim assiège le château du tsar et détruit tout. En revanche, dans la pièce dont Staline est le metteur en scène caché (il ne signa jamais un ordre), c'est la foule des révoltés de la Faim qui est anéantie : le « château », les trains de luxe, les restaurants pompeux pour aristocrates bolcheviques sont indemnes. Staline les offre à ses adulateurs et affidés, lui-même est de mœurs spartiates.

Le petit et antique pays de Galles avait connu la faim, les coups de grisou dans ses mines - rappelons-nous le merveilleux roman de Richard Llewellyn, How Green Was My Valley, paru en 1939, après la mort de Gareth Jones, mais qui célèbre le pays d'où

1 Le titre de la version française est Dans l'ombre de Staline. 
il vient. Un père méthodiste, un village de pêcheurs, humble et chaleureux. On verra le bourg de Barry à la fin du film, quand Gareth, persuadé d'avoir échoué à alerter le monde, revient, par une petite brume, sous les cris ricaneurs des mouettes, dans la maisonnette parentale. Agnieszka Holland nous montre à plusieurs reprises George Orwell tapant fébrilement à la machine son récit Animal Farm. A Fairy story (1945). Comme si ces deux dénonciateurs du raz-de-marée totalitaire qui fond sur l'Europe libérale, Gareth et Orwell étaient liés par un destin secret. C'est une clé de lecture, cachée dans le clair-obscur du film, avec les cochons et autres animaux rebelles qui rêvent d'une égalité impossible. Animal Farm est le texte le plus célèbre qui fournit à l'Europe séduite par Staline, le montagnard à la pipe, un antidote à l'aveuglement de l'utopie. Un aveuglement qui envoya des milliers de jeunes utopistes fanatiques guerroyer en Espagne, comme le fit George Orwell, comme l'aurait fait Gareth Jones s'il n'avait pas été assassiné en Mongolie.

Gareth Jones avait appris le russe à Cambridge parce que sa mère avait été gouvernante des enfants de John James Hughes (1815-1889), l'entrepreneur gallois qui avait fondé le complexe minier et métallurgique de ce qui allait devenir la capitale du Donbass. La ville s'appelait en russe Iouzovka, du nom russifié de son fondateur (Iouz, en transcription phonétique russe), c'est aujourd'hui Donetsk, la capitale d'une des deux républiques séparatistes d'Ukraine orientale. Gareth Jones était doué pour les langues, il parlait sans défaut le russe, le français, l'allemand. Le jeune homme fut présenté à Lloyd George, qui avait été le premier ministre de l'Angleterre en guerre, de 1916 à 1922, dernier premier-ministre libéral (Whig) du Royaume-Uni. Or Lloyd George était d'un village proche de celui de Gareth et il le prit comme secrétaire et comme « plume ». Lloyd George avait perdu le pouvoir avec panache, s'étant en vain opposé à Clémenceau sur la question des réparations imposées à l'Allemagne ; il était persuadé que des réparations trop lourdes seraient fatales : elles feraient disparaître la classe moyenne et prépareraient une dictature dans l'ancien Reich. Gareth partageait le diagnostic de son patron. Il avait obtenu une interview de Hitler en 1930 et il voyait arriver une nouvelle guerre. On le voit dans le film prédire l'événement devant un club de hauts dignitaires anglais fort moqueurs à son égard, mais Lloyd George, au contraire, le convoque et l'engage. Muni d'une lettre de recommandation de l'ancien premier-ministre, il débarque à Moscou. L'ambiance de la Russie soviétique des années 1930, dont certains éléments n'avaient guère changé quand j'y suis moimême arrivé en 1956, était faite de luxe dans les anciens palaces ornés d'immenses portraits de Lénine (l'hôtel Métropole) et de sordides dortoirs où chacun devait lutter pour sa place. Une petite équipe de journalistes occidentaux vit dans le palace, se laisse dicter les informations qu'il convient de répercuter à l'Occident. Tout dans le 
Georges NIVAT - La vérité sur la Grande Famine (Ukraine 1931-1934)

film d'Agnieszka Holland est emprunté aux carnets, aux articles de Gareth et à ceux de ses confrères.

\section{Le film d'Agnieszka Holland}

Les premières scènes annonciatrices nous montrent un Gareth loqueteux et affamé, qui erre en Ukraine et frappe à une porte : "Il y a quelqu'un de vivant ici ? » Ce sont les mots mêmes d'un personnage d'Alexandre Soljenitsyne dans Le premier cercle [В круге первом, 1955-1958], le marxiste fanatique Rubin (alias de l'écrivain Lev Kopelev). Gareth découvrira, et nous avec lui, vers la fin du film que les enfants qui se cachent mangent par petits bouts le corps congelé de Kolja, leur grand frère, étendu derrière la grange...

À Moscou, chez le mieux vu des journalistes étrangers, l'Américain Duranty, c'est le «festin pendant la peste ", une véritable orgie, toujours en un somptueux et inquiétant sépia à la Gainsborough, scènes de bordel pour tous les goûts sexuels, auxquelles se soustrait Gareth, qui, en sortant du lupanar protégé par le KGB, fait la connaissance d'Ada Brooks, une collègue qui va lui révéler le secret de la famine. Gareth n'a pas encore pris sa chambre au somptueux Hôtel Métropole, bâti sous le dernier tsar, en style Belle époque ; il accompagne chez elle Ada, et c'est chez elle qu'il passe la première nuit de ce dernier séjour en URSS. Il y revient pour sa dernière nuit, avant le départ pour le pays de la Grande Famine. Ada lui prépare son sac. Car la décision de Gareth de tout enfreindre et d'aller sur place voir et « vivre » la Famine a séduit Ada, qui lui révèle tout ce qu'elle sait de la catastrophe. Le lendemain de la première nuit, Gareth a déjà commencé son enquête clandestine : il quitte l'hôtel de luxe et se fait admettre dans un minable dortoir où il peut mieux appréhender le gouffre social où le pays de l'utopie est en train de sombrer. Cependant la lettre de Lloyd Georges a fait son effet sur le commissaire du peuple aux Affaires étrangères Maksim Litvinov, et Gareth est emmené en voyage en Ukraine, dont il ne devra toutefois voir, bien sûr, qu'une vitrine.

L'enquête de Gareth commence dans le train luxueux qui l'emmène loin de Moscou. Son amphitryon avale vodka sur vodka, Gareth fait alors mine d'aller aux toilettes, mais ne reviendra pas ; abandonnant sa pelisse fourrée, il a sauté sur la voie, avec le petit sac qu'Ada lui a confectionné avec des miches de pain noir. Lorsqu'il saute dans le convoi d’à-côté, il a changé de monde : des êtres muets, grelottants, affamés, un poêle au milieu du wagon, une indifférence inquiétante. Gareth sort une orange, la mange, en jette la pelure et voit un garçon se précipiter dessus. Le pays de la Famine est devant lui. Il propose à son voisin de lui acheter son manteau. Refus, les billets de banque soviétiques n'ont ici aucune valeur. Un peu plus tard Gareth lui propose du pain ; à ce moment, l'autre se lève, enlève et lui donne son manteau, 
et cache précipitamment la miche sous son chandail en loques. Le train va arriver à Iouzovka, devenu Stalino, Gareth sort. Sous un immense portrait de Staline, une foule peureuse cherche à se faire engager par les soldats du NKVD qui, baïonnette au canon, poussent dans un hangar ceux qu'ils jugent encore assez vaillants pour charger des sacs de grains, confisqués aux paysans, et qui vont partir pour Moscou, et pour l'exportation.

Gareth entre, coltine un premier sac, retourne en prendre un autre avant qu'un assistant soupçonneux ne dise à un soldat que ce type est sûrement un espion. Gareth s'enfuit, rampe sous les wagons voisins, court à perdre haleine sous les balles à travers un bois, tombe, repart, aperçoit une isba. C'est le grand silence de la désolation, c'est l'Ukraine en proie à la Grande Famine. Un loup apparaît, le regarde longuement à trois mètres, hésite, s'éloigne. Il est repu.

Les photos d'Agnieszka Holland sont magnifiques : le noir-et-blanc symbolise qu'on est entré au cœur sinistre de l'histoire. L'enfer est là : ici un couple de vieux paysans allongés, moribonds, là une carriole qui passe, un homme qui jette les corps gelés ramassés au bord du chemin, ou encore une petite fille vivante, accroupie à côté de sa mère morte, et dont les yeux fixent longuement Gareth. Pourtant, pas de pathos, pas d'émotion, plutôt l'enfer d'une glacière humaine. Nous avançons avec Gareth, partageant sa ténacité, son anxiété, sa faim aussi, et c'est peut-être le plus grand mérite du film que de parvenir à nous faire descendre aux enfers en sa compagnie. Il devient notre Virgile et, avec lui, nous entrons dans les mornes entrailles de la Souveraine-Famine. Gareth est finalement arrêté, menotté, menacé, mis au cachot ; mais à Moscou, le KGB a trouvé une parade au risque de voir ébruiter en Occident la famine en Ukraine. On vient d'arrêter six ingénieurs anglais et, à la conférence de presse tenue pour les correspondants attitrés, toujours à l'hôtel Métropole, il est clairement indiqué que les ingénieurs sont des otages. Gareth, libéré et conduit au train sans ménagement, manu militari, devra aller communiquer à Lloyd George les termes du « marché » officieusement proposé.

Gareth ne remplira pas son rôle, au risque de mettre en danger les six ingénieurs britanniques de l'entreprise électrique Metropolitan-Vickers accusés de sabotage. Le film va maintenant nous raconter sa courageuse tentative d'alerter le monde, dénoncer la Famine qui condamne à la mort lente toute une partie de l'Ukraine paysanne. À peine débarqué à Londres, Gareth voit un garçon boucher traverser tranquillement la cohue d'une rue, un quartier de bœuf entier sur l'épaule. Tableau à la Soutine, impensable et insoutenable dans le monde lunaire d'où il vient. Il décide alors de « dire la vérité », et donne dans un grand hôtel une conférence de presse décisive pour démentir les apologies de Duranty et Cie sur l'homme nouveau qu'on est en train de forger au pays des Soviets. Cette décision entraîne des réactions violentes : 
Lloyd George l'accuse d'avoir trahi la confiance mise en lui, son père taciturne lui serre la main sans un mot, et surtout un grand article de Walter Duranty dans le New York Times dénonce la myopie du jeune Gallois. «On ne fait pas d'omelettes sans casser des œufs »; l'URSS souffre ici ou là de mauvais approvisionnement, mais elle ne meurt pas de faim - la transformation de l'homme a son prix. Gareth rentre alors à Barry, son village natal, toutes les portes s'étant fermées pour lui à Londres. Mais dans sa chambrette de Barry, Gareth rédige humblement des articles pour un journal local, le Western Mail, et le premier s'intitule « La faim et l'esclavage » : désormais il n'y a plus de retour en arrière pour Gareth Jones, le voici traître, le voici renégat.

\section{Les voyages au pays de l'utopie}

On est à l'époque des voyages en URSS, au pays de l'utopie. Herbert George Wells, l'immensément célèbre auteur de The Time Machine : An Invention (1895), rencontre Lénine lors de son premier voyage de 1920 - et Staline lors du second, en juillet 1934. Il vient de rencontrer Roosevelt en Amérique ; Staline et Roosevelt lui semblent les deux hommes les plus capables de faire évoluer le monde vers une civilisation socialiste globale, " a Socialist World State ». Dans Experiment in Autobiography (1934), il déclare « n'avoir jamais rencontré un homme plus sincère et honnête » que Staline. "Personne n'a peur de lui et tout le monde a confiance en lui ». C'est en parlant avec Maxime Gorki, après la rencontre avec Staline, que Wells, peut-être jaloux, commence à comprendre qu'il n'y a aucune place pour une opposition en URSS, et particulièrement pas pour un PEN club (Wells en est alors le second président). Sa conclusion est désabusée : « Je m’étais attendu à trouver une Russie nouvelle émergeant de sa torpeur et prête à prendre conscience de Cosmopolis, et je la trouvais sombrant encore plus profond dans le rêve d'opium d'une Soviétie auto-suffisante ». Après Wells, André Gide écrivit son Retour de l'URSS (1936) puis corrigea son texte qui devint Retouches à mon « Retour de l'URSS » (1937) ... Maxime Gorki, qui avait fait le voyage en sens inverse, de Sorrente à Moscou, où on l'avait installé dans l'hôtel particulier de l'ancien industriel russe Pavel Rjabušinskij, y écrivit son célèbre article " Quand l'ennemi ne se rend pas, on l'extermine » [Если враг не сдается, его уничтожают, Pravda, 15 novembre 1930]. On peut y lire :

À l'intérieur de notre pays, et contre nous, sont ligués de très rusés ennemis qui organisent la famine, les koulaks, qui terrorisent les kolkhoziens par le meurtre, l'incendie criminel et autres lâches méfaits. Nous avons contre nous tout ce qui a fait son temps, tout ce qui a été rejeté par l'histoire, pour cette raison, nous sommes en droit de nous estimer toujours en état de guerre civile.

La conclusion s'impose d'elle-même: "quand l'ennemi ne se rend pas, on l'extermine ». L'auteur des Pensées intempestives [Несвоевременные мысли] de 1917-1918, s'opposait violemment à la dictature naissante de Lénine et de Trotski, 
et le voici, dix ans plus tard, qui non seulement se rallie à celle de Staline, mais lance même le mot d'ordre d'extermination de la paysannerie. Il faut dire que Gorki avait toujours considéré le paysan russe comme un illettré brutal, dépourvu de tout altruisme. Alors que la Russie de 1922 était aux prises avec une première famine, Gorki, depuis Berlin où il avait émigré, résumait dans son traité Le paysan russe [О русском крестьянстве, 1922] ce qu'est la paysannerie russe par ce proverbe : « Riazan n’a que faire de la famine à Pskov » [“Не плачут в Рязани о Псковском неурожае"].

«L'ennemi », on l'exterminait bel et bien, mais il n'avait d'ennemi que le nom. Le vieux couple de gisants dans l'isba glacée est la réponse du film d'Agnieszka Holland au slogan ignominieux, hélas, de Gorki, aux faux concoctés par Duranty et Cie saluaient la transformation de l'homme ancien en un « homme nouveau, libre et créateur ». Dans le New York Times, Walter Duranty dénigra avec mépris l'enquête de Gareth Jones. Gareth prend connaissance de l'article, mais comment répondre ? Quelqu'un mentionne dans le couloir du petit canard local de Barry où Gareth a reçu un petit job que le magnat de la presse américaine, un Américain d'une famille originaire de l'Ulster, William Randolph Hearst, est en vacances dans son château à dix kilomètres de là, un château médiéval qu'il a acheté et restauré en 1925. C'est alors que Gareth prend son vélo et fonce au château de Saint Donat's, y pénètre par effraction et convainc Hearst de lui donner une chance. Le New-York Times publiera la réplique de Gareth Jones à Walter Duranty, mais, l'avertit Hearst, ce sera parole contre parole. Une fois de plus Gareth est vaincu par knock-out. Duranty obtint le prix Pulitzer, il est fêté triomphalement à New-York. Le film s'achève sur la jetée de Barry, où Gareth s'enfonce dans la brume. En 2003, des voix demandèrent au jury du Prix Pulitzer de révoquer le Prix attribué en 1933 à Duranty. Le jury refusa.

\section{La vérité chuchotée}

C'est par l'écrivain Vasilij Grossman (1905-1964) que la Famine en Ukraine fut le plus courageusement et magnifiquement décrite, dans son récit Tout passe [Bce течёт]. Naturellement, le récit resta inédit en URSS, comme le roman de Grossman Vie et destin [Жизнь и судьба]. Le titre, venu d'Héraclite, explique pourquoi il ne sert à rien de revenir sur le passé : le mensonge le plus énorme a "coulé », comme l'eau du fleuve, les générations de bourreaux se sont entre-dévorées, et ont souvent été enterrées dans la même fosse commune que leurs victimes. C'est le cas du site de Kommunarka, lieu d'exécution du NKVD de 1937 à 1941, près de Moscou. Et souvent il ne reste que les œuvres d'art pour nous dire les plus insignes cruautés. Ainsi Goya et sa gravure de Saturne dévorant ses enfants, ou son tableau du « Trois mai ». Tout passe de Grossman est un ouvrage lumineux, sans doute le plus lumineux 
de tout le $\mathrm{XX}^{\mathrm{e}}$ siècle en toutes langues. Les noirceurs du siècle, les cruautés aveugles enfantées par le fanatisme, la délation, la malveillance ou la foi aveugle dans le Guide suprême, la déshumanisation et le stupre du camp y sont nommés et révélés dans une inoubliable conversation nocturne entre un homme revenu du camp et une femme qui s'est éprise de lui et qui a vécu la grande famine. Confession chuchotée et réciproque où la jeune fanatique qui était convaincue de forger l'Avenir radieux en éradiquant les koulaks raconte l'enfer de la Grande Famine au rescapé d'un autre enfer, le Goulag. Leurs chuchotements nocturnes pénètrent en nous comme une confidence mystérieuse et interdite : les étapes de la mort par la faim, les enfants aux têtes énormes, aux cous de cigogne, aux ventres ballonnés, " qui ont l'air d'avoir été sur cette terre depuis plus de soixante-dix ans », écrit Grossman, les fantômes rampant vers les voies ferrées où les trains sont munis de volets pour cacher aux voyageurs la vermine humaine en quête de miettes ou d'épluchures, puis le retour des fantômes dans leur maison-cercueil :

Les gens étaient couchés sur le poêle ou des lits, certains respiraient encore, d'autres plus ; la fille de la maison, devenue folle, rongeait le pied d'un tabouret. Quand je suis entrée, elle ne s'est pas même retournée, elle s'est mise à grogner comme un chien dont on s'approche, en train de ronger un os. La famine était totale, la mort frappa. D’abord enfants et vieillards, puis les personnes d'âge moyen. On cessa d'enterrer. Les derniers à mourir sont restés couchés dans leur isba. Le silence se fit. Tout le village mourut. Je ne sais qui fut le dernier. Nous autres, qui travaillions à l'administration, on nous a ramenés à la ville (Grossman 1984, 203).

Ces pages de Vasilij Grossman sont sidérantes : le cannibalisme, moins de l'autre que de soi (le corps s'auto-dévore), la déshumanisation de l'autre et, malgré tout, ce regard enveloppant, cette bienveillance ultime de l'Écrivain-Juge. Et qui affirme doucement : le bourreau perd son humanité, la victime la conserve - même l'enfant au cou de cigogne, vieux de soixante-dix ans... Car Grossman a tout vu, tout jugé, et pris par la main un monde entier de mourants au ralenti.

Tu as vu dans les journaux les enfants des camps allemands ? C'était exactement la même chose. Une tête comme un boulet de canon, un cou de cigogne, et cette peau tendre sur leur squelette comme une gaze jaune (ibid., 199).

Grossman n'eut pas besoin de Hannah Arendt pour comparer les deux totalitarismes du siècle. Lui-même ne fut pas déporté, il alla avec l'écrivain Il'ja Erenburg (18911967) découvrir Treblinka, leur Livre noir [Чёрная книга 1943-1945] fut interdit... Il s'enferma dans la solitude et l'écriture clandestine. « L'enfermement l'a fait sombrer dans le mysticisme », aurait déclaré Mihail Suslov en 1962 en prenant connaissance de son récit La Madone Sixtine [Сикстинская Мадонна, 1986].

Les ouvrages des historiens après Grossman sont venus, en quelque sorte, confirmer son regard omniscient. Bien sûr, grâce à Gareth Jones, on pouvait, dès 1933, savoir avec précision. En 1935, Boris Souvarine, marxiste oppositionnel, fils 
d'une famille juive karaïte d'Ukraine, avait publié à Paris, à petit tirage, chez Plon, son Staline, Aperçu historique du bolchevisme. Avec la précision d'un Tacite dénonçant les Césars et la perspicacité d'un Custine affirmant «ici mentir, c'est protéger la société », il y brosse en 600 pages un extraordinaire panorama du pays confisqué par Staline, de l'asservissement progressif des esprits, des mécanismes du mensonge. Souvarine écrit à Paris, il ne dispose que de peu de témoignages ; néanmoins son diagnostic est tranchant. Lénine pensait qu'il fallait un long travail d'éducation avant de pouvoir imaginer « un travail agricole en commun ». Staline n'en a cure. Souvarine parle des «villages entiers, des cantons, des districts dépeuplés, leurs habitants dispersés et décimés, comme autrefois en Assyrie et en Chaldée » (Souvarine 1935, 480). Le lexique de Souvarine est précis, emprunté aux codes et lois venus du droit romain - « le consentement des intéressés, écrit-il, n'est atteint, par l'expropriation et le pillage illégal, qu'au prix d'une répression sans exemple, que Staline intitule "suppression du koulak comme classe" " (ibid.). Souvarine dispose des calculs de Hugh Walpole, qui a scruté les données du commissariat du Travail et publié des lettres de déportés mennonites chassés d'Ukraine [Out of the Deep, Letters from Soviet Timber Camps, 1933], et de Ivan Solonevič, un des rares évadés du bagne soviétique $^{2}$. Le chiffrage se fait entre cinq et dix millions de victimes. Souvarine ne parle pas d'un traitement spécifique à l'Ukraine ; l'agriculteur-prolétaire, tout comme l'ouvrier-dictateur sont également réduits en servage. Il cite Flaubert, qui se fait du socialisme l'idée d'une sorte de "Lacédémone américaine, où l'individu n'existerait que pour servir la société plus omnipotente, absolue, infaillible et divine que les Grands Lamas et les Nabuchodonosors » (ibid., 493). Pour faire comprendre la nouvelle dictature, Souvarine et bien d'autres (Nadežda Mandelstam, Pierre Pascal) ont recours à l'antiquité orientale...

\section{Robert Conquest, Sanglantes moissons}

Mais après ces précurseurs, il fallut attendre le livre de l'historien américain Robert Conquest (1917-2015) pour avoir le premier ouvrage scientifique sur la Famine de 1933, Harvest of Sorrow, Soviet Collectivisation and the Terror-Famine (1986).

Cinquante ans avant que je n'écrive ces lignes, l'Ukraine et les Ukrainiens, les Cosaques et les habitants d'autres régions plus à l'Est, soit un territoire de plus de quarante millions d'habitants - ressemblait à un immense camp Belsen-Belsen ${ }^{3}$. Un quart de million de la population rurale, hommes, femmes, enfants gisaient, morts ou mourants, les autres étaient à des degrés divers de débilité, sans la force d'enterrer leur famille ou voisins (Conquest 1986, 3).

2 Ivan Solonevič, La Russie en camp de concentration [Россия в концлагере]), première publication en 118 feuilletons dans le quotidien russe Poslednie Novosti [Les dernières nouvelles], Paris, 1935-1936.

3 Le camp de concentration en Saxonie où mourut Anne Franck. 
Dans son introduction, Robert Conquest se demande comment il se fait que pareils événements ne soient pas encore complètement enregistrés dans notre conscience publique. C'était avant l'indépendance de l'Ukraine, qui a fait suite à l'écroulement de l'Union soviétique. Aujourd'hui, la Grande Famine est étudiée en Ukraine, elle y est même devenue un événement fondateur pour la conscience de ce pays.

Mais ce déficit d'attention à la famine de 1933 vaut pour toute la Russie, et également pour bien des études occidentales sur l'URSS. Non seulement cette spécificité n’était pas mentionnée et ne figurait pas dans la plupart des ouvrages qui analysaient la structure de l'agriculture soviétique, mais même la famine était à peine mentionnée. J'ai étudié en 1955 à la Sorbonne, nous lisions le livre du père Henri Chambre, Le marxisme en Union soviétique : idéologie et institutions (1955). La propriété collective en URSS y était étudiée comme on étudie les preuves de l'existence de Dieu, en dehors de toute référence à la réalité. En s'appuyant sur les derniers textes de Staline, le père jésuite étudie le rôle de la superstructure dans l'idéologie et la praxis soviétique, et l'adéquation nouvelle entre conscience et praxis révolutionnaires. Douze après, en 1967, dans un livre intitulé Union soviétique et développement économique, le père Chambre évoque - mais seulement en quelques lignes - «des méthodes extrêmement brutales qui ont pesé lourdement sur le développement ultérieur de l'agriculture soviétique » (Chambre 1967, 223). Il fallut donc attendre l'ouvrage de Robert Conquest pour sortir de cette «théologie » de la soviétologie et avoir une tentative historienne plus proche du réel et de ses souffrances indicibles.

Robert Conquest part de l'analyse de la classe paysanne par Marx et le marxisme. Le Manifeste du Parti communiste de 1848 demande l'abolition de la propriété de la terre ; la collectivisation en URSS devait mener le monde rural à une concentration des moyens qui ferait de l'agriculture une sorte de travail industriel, peu différencié du travail ouvrier. Les activistes de la dékoulakisation étaient persuadés d'œuvrer dans le sens de l'histoire, en marche vers l'utopie finale, qui serait un anarchisme total, alors qu'on avait pour l'instant une dictature d'une extrême violence. Certes, en 1986, les témoignages avérés manquaient encore pour la rédaction du livre. Conquest fait appel, par exemple, à Mihail Šolohov (1905-1984), qui a décrit avec beaucoup de cruauté les excès de la collectivisation dans Terres défrichées [Поднятная целина, 1932-1959]. En avril 1933, Šolohov écrit à Staline une lettre pour lui signaler la brutalité de la collectivisation dans la région du Don, d'où il est originaire. Des communistes ou des kolkhoziens sont venus lui demander d'intervenir. Ils dénoncent une «méthode systématique » d'humiliation et de terreur. Staline répondit à son ami Šolohov que ses mots donnaient une «impression de partialité »; en fait, les « honorables producteurs de grain de votre district conduisent une grève à l'ítalienne 
(sabotage), et n'ont pas hésité à priver de grain l'Armée rouge et nos ouvriers » (cité par Conquest 1986, 233). Conquest s'appuie habilement sur une petite partie de la littérature officielle, et aussi sur quelques récits de dissidents comme ceux de Leonid Pljušč (1939-2015). La chronique que fait Conquest des articles de la Pravda, appelant à la lutte impitoyable contre les koulaks est très parlante. Conquest apporte aussi des témoignages de survivants, très jeunes à l'époque des faits, qui ont émigré en Occident, mais en nombre limité. Sur le problème de la spécificité du « génocide ukrainien ", l'historien est prudent. Pour lui, c'est l'idéologie communiste avant tout qui a déclenché un massacre sans précédent par la famine en Ukraine.

\section{Anne Applebaum, La famine rouge}

Toute autre est l'approche d'Anne Applebaum dans son dernier livre Red Famine: Stalin's War on Ukraine, paru en 2017. Les archives se sont ouvertes, des témoins nouveaux ont eu le temps de parler, l'Ukraine est indépendante, mais elle est en « guerre hybride » avec la Russie du président Vladimir Poutine, qui a repris la Crimée donnée par Nikita Khrouchtchev à l'Ukraine soviétique en 1954 et qui soutient les deux « républiques » séparatistes du Donbass, celle de Louhansk et celle de Donetsk. Ce même Donetsk fondé par l'homme d'affaires gallois John James Hughes et qui s'appela successivement Iouzovka, puis, de 1924 à 1961, Stalino, avant de recevoir son nom actuel, Donetsk. De l'œuvre de Hughes il reste un immense ensemble minier et métallurgique, qui faisait de Donetsk la capitale industrielle de l'Ukraine avant 1994 - mais qui vit aujourd'hui séparée du reste du pays ${ }^{4}$. Anne Applebaum consacre à Gareth Jones trois pages vers la fin de son ouvrage - c'est mieux que Conquest, qui lui accorde dix lignes. De Conquest à Applebaum on est passé d'une vision à une autre. Car Anne Applebaum épouse fortement la cause ukrainienne, voit la Russie comme la puissance colonisatrice qui a occupé l'Ukraine pendant plus de trois siècles, et organisé un génocide par la famine. Pour Robert Conquest, c'était l'idéologie marxiste, manipulée par Staline et véhiculée par de jeunes fanatiques qui avait enclenché la famine ; pour Applebaum le caractère génocidaire anti-ukrainien ne fait pas de doute, même si le mot de « génocide » n'est finalement pas retenu en raison des réserves des juristes.

Les nouveaux aperçus qu'apporte Anne Applebaum sur cette Terreur par la Famine sont saisissants, et même certains très durs à lire ; ils forment le cœur de l'ouvrage. Mais ce cœur est précédé par de longs chapitres introductifs et suivi d'un long épilogue où elle discute du déni et du mythe. On peut être gêné par certains anachronismes, comme par exemple le terme de «dirigeants coloniaux» en

4 On trouve dans les remarquables Ukrainische Lektionen de Karl Schlögel une histoire de la ville et de Hughes meilleure que tout ce que je pourrais ici résumer. 
parlant du XIX ${ }^{\mathrm{e}}$ siècle. Dans l'Empire russe, on appelait « colons » les nombreux étrangers (italiens, souabes, écossais, néerlandais, français, etc.) auxquels il était fait appel, en particulier sous la Grande Catherine. Le Gallois Hughes en est un exemple, quoique à un niveau plus haut, la majorité étant des artisans. Les Cosaques, eux, venaient d'un peu partout et gardaient les «marches » de la Russie, le mot Ukraine ayant d'ailleurs ce sens de "marche» ou de «limite». En 1670, l'hetman Bohdan Hmelnitsky prêta serment au tsar à Pereïaslav et fit prêter serment à son peuple devant les ambassadeurs du tsar selon la formule « Le tsar a ordonné, les boyards ont décidé ». Il s'agissait d'avoir la protection de l'autocrate russe orthodoxe contre les seigneurs polonais catholiques. Bien entendu, l'Ukraine devint une sorte de province russe, la Petite-Russie, province qui donna à l'empire de grands serviteurs et où le servage fut beaucoup moins répandu qu'en Russie. Nikolaj Kostomarov (1817-1885), nationaliste ukrainien (fondateur de la « Fraternité Cyrille et Méthode ») et historien russe, définit de façon précise et imagée le différentiel entre Ukraine et Russie dans un texte qui garde toute son actualité, Deux nationalités russes [Две русские народности, 1861] - l'une fondée sur l'amour de la liberté,l'autre sur Dieu et le tsar, l'une aimant les fleurs, l'autre n'aimant pas la nature... L'Ukraine a joué pour l'intelligentsia russe le rôle de l'Italie pour les Allemands, Goethe en particulier. L'histoire de l'Ukraine a été écrite essentiellement par des historiens de la diaspora ukrainienne - au Canada et aux États-Unis - à Harvard, où se trouve le plus important Centre d'études ukrainiennes. Mais le flambeau a aujourd'hui été repris à Kiev et Kharkov. L'historien Miroslav Popovič (1930-2018) a élaboré dans son Histoire de la culture ukrainienne [Нарис історії культури України, 1998] се qui est peut-être la plus éclairante conception de l'Ukraine, à partir de la notion de baroque.

Anne Applebaum donne en raccourci une idée du chaos dans lequel fut plongée l'Ukraine en 1917 et de la succession des Allemands et de Pavlo Skoropadsky, des bolcheviks (avec les réquisitions alimentaires - prodrazviorstka), et de l'anarchiste Nestor Makhno, qui veut un "socialisme sans le bolchevisme ». Les réquisitionspillages concernent l'Ukraine, mais aussi la Russie, le Kouban, le Tatarstan. Chaque région mériterait une étude à elle seule. Par exemple, la Famine réapparaît le long de la Volga. Elle avait déjà sévi en 1891-1892, et Tolstoï et sa famille s'étaient alors mobilisés. Elle sévit à nouveau en 1921-1922, et c'est Maxime Gorki qui lance alors un appel à l'aide. Applebaum nous fait suivre les événements à l'aide de quelques témoins récurrents. On voit la collectivisation débuter dans le village du paysan Miron Dolot, dont l'auteure cite à plusieurs reprises le journal, afin que nous sentions bien la montée de la terreur et de la famine. Les chiffres des victimes, nous dit Applebaum, sont toujours plus importants en Ukraine qu'ailleurs. La cruauté se déchaîne au début 
des deux côtés. Un activiste qui, le soir, a fait le clown dans l'église avec les habits du prêtre est retrouvé mort le lendemain. " Nous pourrions perdre l'Ukraine ", écrit Staline à Lazar Kaganovič (Applebaum 2017, 199). On exporte les céréales, alors que la famine commence.

Les détails les plus étonnants détails que donne Anne Applebaum portent sur la soumission des dirigeants du Parti communiste ukrainien qui savent très bien qu'il n'y a plus de grain à réquisitionner, plus de bétail à prendre pour le kolkhoze, plus de patates cachées nulle part. Staline soumet Stanislav Kossior et les autres dirigeants du Parti communiste d'Ukraine à de nombreux ultimatums. Ils cèdent, bien sûr, mais, aux échelons inférieurs, l'historienne nous montre la résistance, silencieuse et parfois héroïque, de chefs bolcheviks locaux. À Odessa, les dockers refusent de charger les bateaux étrangers. L'établissement des listes de koulaks à déporter, listage des victimes ainsi condamnées, donne lieu à des milliers de drames, où vilénie, vengeance, jalousie et parfois noblesse de cœur s'emmêlent. L'historien Myhajlo Hruševksy (1866-1934), ancien premier président de la République d'Ukraine en 1917-1918, est à présent soupçonné d'«idéologie bourgeoise ». Quarante bibliothécaires de la Bibliothèque nationale à Kiev sont accusés de "national-fascisme », et arrêtés ; soixante-neuf églises de Kiev sont détruites... L'ukrainisation menée officiellement pendant les années 1920 devient un chef d'accusation. Les villages mourants sont interdits d'accès, le passeport interne est introduit en décembre 1931, rétablissant de facto un servage que l'Ukraine avait moins connu que la Russie centrale. Le chapitre de l'ouvrage d'Anne Applebaum consacré aux activistes qui débarquent pour confisquer, fouetter les enfants qui glanent, surveiller les cheminées qui fument le soir, saisir sans fin et sans aucun profit, est l'un des plus terribles. C'est l'année où l'écrivain Il'ja Erenburg décide de rentrer en URSS et de se mettre au service de l'idéologie stalinienne. Bientôt, il va partir observer la construction de la magistrale Moscou-Don puis du complexe industriel de Kouznetsk, en Sibérie. Il en sortira le roman Le deuxième jour [День второй, 1933], comme si Staline refaisait l'Euvre du Créateur, comme Dieu dans le livre de la Genèse, d'où est tirée la citation en exergue du roman : « Et il y eut un soir, et il y eut un matin : deuxième jour ». Le Deuxième jour était pour l'industrialisation ce que Terres déchiffrées de Mihail Šolohov était pour la collectivisation. Erenburg se rendit sur les lieux de la construction du combinat de Kouznetsk, où travaillaient 220000 hommes, rencontra le directeur, qui ne dormait presque pas, mangeait en se déplaçant, un homme absolument possédé par sa mission ce qui ne l'empêcha pas d'être fusillé cinq ans plus tard ${ }^{5}$. Pas plus que Šolohov, Erenburg ne cachait la rudesse, la cruauté de cette nouvelle Genèse du monde qu'applaudissaient Romain

5 Je me réfère ici à l'ouvrage de Boris Frezinskij consacré à Il’ja Erenburg, véritable somme historique et monumentale, pleine de sympathie pour l'auteur, sans sacrifier aucunement la vérité (Борис Фрезинский, Об Илье Эренбурге (книги, люди, страны), Москва, Новое литературное обозрение, 2013). 
Rolland, André Malraux, André Gide et tant d'autres à l'Ouest. Mais pour forger l'homme nouveau, ne fallait-il pas de la rudesse, voire une famine éducatrice ? Car on aurait pu imaginer un troisième type de roman éducateur, celui de la Famine ! Mais la littérature soviétique n'eut pas le temps d'y parvenir, les purges commencèrent. En somme, il fallut attendre Vasilij Grossman... L'année 1931 est l'année du suicide de Vladimir Majakovskij, 1932 est celle du suicide de Nadežda Allilueva, la femme de Staline. Le médecin dut réviser son premier certificat de décès. Tout le Parti chuchota que c'était l'ultime acte de protestation de Nadežda face à la famine ; sa fille Svetlana, dans ses Mémoires, parle de la terrible désillusion de sa mère à l'égard de son père.

Tournons-nous à nouveau vers Anne Applebaum: elle donne une analyse saisissante de la « mutilation des émotions », de ce qu'est la « pensée de la faim », avec disparition des sentiments familiaux, hallucinations, lynchages, silence final total, puanteur terrible. Russes et Biélorusses envoyés pour s'établir dans les chaumières des victimes en repartent aussitôt, tant la puanteur est indestructible. L'excédent de morts en 1932 sur l'année 1931 était officiellement, écrit Applebaum, de $3 \%$ en Russie et de presque 15 \% en Ukraine. En 1937, Khrouchtchev arrive pour purger le pays, Stanislav Kossior et les autres dirigeants du Parti sont exécutés. Une race nouvelle d' « hommes instruits, sensés, actifs » est en train de naitre, avait prophétisé Gorki dans Le paysan russe...

La suite, c'est le Holodomor dans l'histoire. La Wehrmacht tout d'abord fêtée à son entrée en Ukraine, puis le collaborationnisme, puis le "Plan de la faim » de Hitler, miroir symétrique de la famine organisée par Staline. La Vérité, que cherchait Gareth Jones, n'était pas près d'apparaître. Mais après la défaite allemande, l'un des innombrables $D P^{6}$ ukrainiens qui ont fui avec l'armée allemande, l'ethnographe Oleksa Voropaj publie à Londres en 1953 Dans le neuvième cercle... [В Дев’ятім Крузі...], ouvrage traduit en 1983 par le Centre d'Études ukrainiennes de Harvard sous le titre The Ninth Circle. Scenes from the Hunger tragedy of Ukraine in 1933, avec trente ans de retard! Mais en 1987, survient la réplique à Voropaj, surprenante à bien des égards. Elle est due à Douglas Tottle, un syndicaliste canadien : Fraud, Famine, and Fascism, The Ukrainian Genocide : Myth from Hitler to Harvard. « Fraude, Famine, Fascisme - le génocide ukrainien ou le mythe de Hitler à Harvard », en somme les trois grands « $\mathrm{F}$ » concoctés par deux grands « $\mathrm{H}$ », ou l'idée que Hitler a des acolytes à Harvard... L'argumentaire est celui des copeaux qui volent quand on abat un arbre, ou des œufs qu'on casse pour faire l'omelette (comme dans l'article de Duranty). Oui, on a souffert de la faim, mais la modernisation l'exigeait, et les intempéries s'en sont mêlées - plaide l'auteur, parfaitement manipulé.

6 Displaced Person. 


\section{Juger ou ne pas juger}

Écrire l'histoire de pareilles catastrophes humaines sans formuler de jugement est presque impossible. Mais la définition du crime est en soi un problème. Et ici, il convient de laisser la parole à l'historienne Applebaum. « Staline, on l'a vu, ne chercha pas à tuer tous les Ukrainiens, pas plus que tous les Ukrainiens ne résistèrent » (Applebaum 2017, 404). Le mot " génocide », nous rappelle-t-elle, fut inventé par le juriste Raphael Lemkin de la ville de Lviv (Lvov, Lwów, Lemberg) dans un livre intitulé Axis Rule in Occupied Europe, publié en Amérique en 1944. Applebaum admet que le Holodomor ne répond pas aux critères de Lemkin et du procès de Nuremberg. Staline ne nourrissait sans doute pas de haine particulière à l'égard de l'Ukraine, il manipulait l'idéologie marxiste, et il envoûta des dizaines de milliers de jeunes fanatiques qui devinrent des bourreaux du paysan des Terres Noires de l'Ukraine qui avaient été le "grenier de l'Europe ». Mais ces jeunes bourreaux ont sévi ailleurs, et par exemple on les voit à l'œuvre au Tatarstan dans le roman de Güzel' Jahina Zouleïkha ouvre les yeux... On ne peut vraiment aborder le problème du Holodomor et du Grand mensonge qui l'accompagne sans prendre en compte ce que, depuis l'Archipel du Goulag [Архипелаг ГУЛАГ, 1958-1967] de Soljenitsyne, l'on appelle le Goulag et qui occupe l'immensité de la carte géographique de la Russie.

Le problème du chiffrage des victimes du Goulag est étudié aujourd'hui par les historiens dans les archives alors qu'auparavant on le supputait par comparaison, en évaluant les « trous » démographiques, et en recherchant des témoignages partiels. C'est ce que font des historiens russes, dont les recherches ont commencé avec l'ouverture des archives en Russie, et qui posent la question : "Qui et combien ? ". C'est ce que fait l'historien Oleg Hlevnjuk, de livre en livre, et d'article en article. Ses calculs aboutissent à environ un million de fusillés, dix-sept millions de détenus, six millions de déportés.

Ivan Karamazov déclare à son frère, le moine Aliocha, que tant qu'il restera dans l'univers une petite fille qui souffre et qui pleure, il « rend son billet à Dieu ». Même si les statistiques ne sont pas tout, le billet d'un Ivan Karamazov de 1953, à la mort de Staline, aurait été le même chiffre puissance trente millions ! "Si Dieu n'existe pas, qui donc les entendra? », semble répondre Vasilij Grossman dans le murmure nocturne de Tout passe. 


\section{Références bibliographiques}

\section{Oeuvres citées}

Ehrenbourg, Ilya. 1933. Le deuxième jour [День второй]. Paris : Gallimard.

Ehrenbourg, Ilya, et Vassili Grossman. 1995 [Première publication en russe 1993]. Le Livre noir [Чёрная книга]. Arles : Actes Sud.

Gide, André. 1936. Retour de l'URSS. Paris : Gallimard.

Gide, André. 1937. Retouche à mon «Retour de l'URSS ». Paris : Gallimard.

Grossman, Vassili. 1980. Vie et destin [Жизнь и судьба]. Lausanne : L'Âge d'Homme.

Grossman, Vassili. 1984. Tout passe [Все течёт]. Lausanne : Julliard /L'Âge d'Homme.

Gorki, Maxime. 1975. Pensées intempestives [Несвоевременные мысли]. Lausanne : L’Âge d'Homme.

Iakhina, Gouzel. 2017. Zouleikha ouvre les yeux [Зулейха открывает глаза]. Paris : Noir sur Blanc.

Siriol Colley, Margaret. 1999. Gareth Jones : The Manchukuo Incident. Nigel Linsan Colley.

Souvarine, Boris. 1935. Staline. Aperçu historique du bolchevisme. Paris : Plon.

Tottle, Douglas. 1987. Fraud, Famine and Fascism: The Ukrainian genocide myth from Hitler to Harvard. Toronto : Progress Books.

Андреев, Л. Н. 1908. Царь-Голод [Notre Souveraine la Famine]. СПб : Шиповник.

Горький, М. 1922. О русском крестьянстве [Le paysan russe]. Берлин : Издательство И. П. Ладыжникова.

Гроссман, В., и И. Эренбург. 1993 [год написания : 1946]. Чёрная книга [Le Livre Noir]. Вильнюс : Тарбут.

Костомаров Н. 1861. « Две русские народности [Deux nationalités russes] ». Основа. № 3 C. 33-80.

Солженицын, А. 1968 [год написания : 1955-1958]. В круге первом [Le premier cercle]. Evanston : Harper \& Row.

Солоневич, И. « Россия в концлагере " [La Russie en camp de concentration]. Последние новости, 1935-1936.

Солженицын, А. 1973 [год написания : 1958-1968]. Архипелаг ГУЛАГ [L'archipel du Goulag]. Париж : ИМКА-Пресс.

Шолохов, М. А. 1932-1959. Поднятная целина [Terres défrichées]. Москва : Гослитиздат. Эренбург, И. 1933. День второй [Le deuxième jour]. Москва : ГИХЛ.

Воропай О. 1953. В Дев’ятім Крузі... [Dans le neuvième cercle...]. Лондон : СУМ.

Попович, М. 1998. Нарис історї культури Украӥни [Histoire de la culture ukrainienne].

Kiev : АртE 
Travaux académiques

Applebaum, Anne. 2017. Red Famine: Staline's War on Ukraine. New-York: Doubleday.

Chambre, Henri.1955. Le marxisme en Union Soviétique. Paris : Seuil.

Chambre, Henri. 1967. Union soviétique et développement économique. Paris : AubierMontaigne.

Conquest, Robert. 1986. Harvest of Sorrow, Soviet Collectivization and the Terror-Famine. Oxford: Oxford University Press (traduction française : Sanglantes moissons, Paris, Robert Laffont, 1995).

Dmytrychyn, Iryna, éd. 2016. La grande famine en Ukraine. Holodomor, 1932-1933. Connaissance et reconnaissance. Paris : L'Harmattan.

Hlevnjuk, Oleg. 2004. The History of the Gulag, From Collectivization to the Great Terror. New Haven, CT: Yale University Press.

Hlevnjuk, Oleg. 2017. Staline. Paris : Belin.

Hrynevych, Liudmyla. 2021. "Stalin's Faminogenic Policies in Ukraine: The Imperial Discourse ». East/West: Journal of Ukrainian Studies VIII (1): 99-143.

Lemkin, Raphäl. 1944. Axis Rule in Occupied Europe. Washington: Carnegie Endowment for International Peace.

Snyder Timothy. 2012. Terres de sang. L'Europe entre Hitler et Staline. Paris : Gallimard (première édition en anglais en 2010).

Sokoloff, Georges. 2000. 1933. L'année noire. Témoignages sur la famine en Ukraine. Paris : Albin Michel.

Walpole, Hugh. 1933. Out of the Deep, Letters from Soviet Timber Camps. London: Geoffrey Bles Ltd.

Werth, Nicolas. 2020. Les grandes famines soviétiques. Paris : PUF.

Open Access Publications - Bibliothèque de l'Université de Genève Creative Commons Licence 4.0

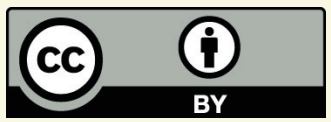

